\title{
Aferição do tempo e do custo médio do trabalho da enfermeira na consulta de enfermagem*
}

\author{
TIME AND MEAN COST RATE OF ANURSE'S WORK IN NURSING CONSULTATION \\ EVALUACIÓN DEL TIEMPO Y DEL COSTO MEDIO DEL TRABAJO DE LA ENFERMERA \\ EN LA CONSULTA DE ENFERMERÍA
}

\section{Elisabete Sabetta Margarido', Valéria Castilho²}

\section{RESUMO}

Neste estudo, a autora objetivou caracterizar os atendimentos de Consulta de Enfermagem, segundo pacientes pré-cirúrgicos do Programa de Coronária, estimar o tempo e o custo médio do trabalho de enfermeira na realização da Consulta de Enfermagem, estudar a existência de associação/correlação entre o tempo, o custo e outras variáveis de interesse. $\mathrm{O}$ desenvolvimento da consulta seguiu as fases da Sistematização da Assistência de Enfermagem (SAE). A amostra foi constituída de 44 pacientes, sendo que 37 (84\%) foram atendidos em consultas novas e $7(16 \%)$ em consultas de seguimento. Os resultados alcançados foram: tempo médio igual a 48,91 minutos para as consultas novas e 22,14 minutos para as de seguimento, com um custo médio de cerca de $\mathrm{R} \$$ 18,01 para as consultas novas e de $\mathrm{R} \$ 8,15$ para as de seguimento. Os resultados mostram que o tempo médio de consultas novas superam de forma estatisticamente significativa o tempo médio das consultas de seguimento e, conseqüentemente, os custos.

\section{DESCRITORES}

Gerenciamento do tempo. Enfermagem.

Custos e análise de custo.

\begin{abstract}
In this study, the author aimed at characterizing Nursing Consultation attendances according to presurgical patients of the Coronary Program, estimating the nurse's average time and work mean cost in performing it, and studying the existence of association/ correlation between time, cost and other variables of interest. The development of the consultation followed the phases of the Assistance Systemization Nursing (ASN). The sample was comprised of 44 patients, of which $37(84 \%)$ were attended in new consultations and $7(16 \%)$ in follow up consultations. Results obtained from the coronary program were mean time of 48.91 minutes for new consultations and 22.14 minutes for follow ups, with a mean cost of around $\mathrm{R} \$$ 18.01 for new consultations and $\mathrm{R} \$ 8.15$ for follow ups. These figures show that new consultations mean time exceeds with statistical significance follow up consultations mean time and, consequently, so do costs.
\end{abstract}

\section{KEY WORDS}

Time management.

Nursing.

Costs and cost analysis.

\section{RESUMEN}

Este estudio tuvo como objetivo caracterizar las atenciones de la Consulta de Enfermería según los pacientes pre quirúrgicos del Programa de Coronaria, estimar el tiempo y el costo medio del trabajo de la enfermera en la realización de la Consulta de Enfermería, analizar la existencia de asociación/correlación entre el tiempo, el costo y otras variables de interés. El desarrollo de la Consulta de Enfermería siguió las fases de la Sistematización de la Asistencia de Enfermería (SAE). La muestra incluyó 44 pacientes, $37(84 \%)$ fueron atendidos en nuevas consultas y 7 (16\%) en consultas de seguimiento. Los resultados alcanzados fueron: tiempo medio de 48,91 minutos para las nuevas consultas y de 22,14 minutos para las de seguimiento, con un costo medio de aproximadamente $\mathrm{R} \$ 18,01$ para las nuevas consultas y $\mathrm{R} \$$ 8,15 para las de seguimiento. Los resultados muestran que el tiempo medio de nuevas consultas supera de forma estadísticamente significativa el tiempo medio de las consultas de seguimiento y, consecuentemente, los costos.

\section{DESCRIPTORES}

Administración del tiempo.

Enfermería.

Costos y análisis de costo.

\footnotetext{
* Extraído da

Dissertação "Aferição do Tempo e do Custo Médio do Trabalho da Enfermeira na Consulta de Enfermagem em Ambulatório de Cardiologia", Escola de Enfermagem da Universidade de São Paulo (EEUSP), 2004.

1 Enfermeira Chefe do Ambulatório do Instituto do Coração - HCFMUSP. Mestre em Administração de Serviços de Enfermagem - EEUSP Vice Coordenadora do Curso de Especialização em Gerenciamento em Enfermagem da Universidade Bandeirantes UNIBAN.

enfbete@incor.usp.br. 2 Enfermeira. Doutora em Enfermagem. Docente do Departamento de Orientação Profissional da EEUSP.

valeriac@usp.br
} 


\section{INTRODUÇÃO}

Atualmente, as enfermeiras, no cotidiano de suas atividades gerenciais começam a se preocupar em obter informações sobre custos. Dessa forma, podem fundamentar seus argumentos em relação à obtenção e manutenção de recursos para a assistência de enfermagem e, além disso, conhecer os custos reais do seu trabalho.

Questões relativas a custos são importantes dentro das instituições de saúde, públicas ou privadas, pois estas freqüentemente contam com recursos escassos.

Com a realização da Consulta de Enfermagem em nossa instituição para os pacientes do Sistema Único de Saúde (SUS) e com a possibilidade desse atendimento ser aplicado também à pacientes particulares e conveniados, originou a questão a respeito do tempo necessário que deve ser reservado para esse atendimento e do custo deste procedimento.

Esta questão nos impulsionou a desenvolver este estudo, dentro de uma perspectiva econômica.

A avaliação econômica é uma análise sistemática que facilita a identificação de alternativas para a introdução de programas de saúde com o conhecimento da magnitude dos problemas. Esclarece que a análise econômica lida com insumos e produtos que são os custos e com as conseqüências das ações, sendo que a análise dessa relação custos e resultados auxilia nas tomadas de decisões ${ }^{(1)}$.

Uma dificuldade reconhecida na realização da avaliação econômica, diz respeito a necessidade de se calcular o custo unitário das atividades ou procedimentos que compõem um programa de assistência, como é o caso da Consulta de Enfermagem.

Custo é um gasto, que só é reconhecido como custo no momento da utilização dos fatores de produção (bens e serviços) para a fabricação de um produto ou execução de um serviço $^{(2)}$.

O conhecimento dos custos nas organizações de saúde e na enfermagem têm como principais objetivos: avaliar o crescimento quantitativo e financeiro das unidades, conhecer os custos dos tratamentos e procedimentos realizados, negociar a tabela de preços e remuneração, alocar os recursos de modo eficiente, identificar ineficiências e desperdícios no processo produtivo, entre outros.

Em relação ao tempo no trabalho, ele

se constitui em um dos recursos fundamentais de uma organização, uma vez que a sua gestão contribui para a melhoria dos desempenhos coletivo e individual e, conseqüentemente, da produtividade ${ }^{(3)}$.

As principais finalidades do estudo do tempo são: estabelecer padrões para os processos de produção, fornecer dados para determinar o custo padrão, estimar o custo de um produto ou serviço novo e fornecer dados para o estudo do balanceamento de estruturas de produção ${ }^{(3)}$.

O padrão de mão de obra é definido como a quantidade de minutos que o trabalhador utiliza para concluir um item do trabalho, sob condições normais ${ }^{(4)}$.

Vários métodos têm sido aplicados para estudar o tempo no trabalho, sendo possível adotar os mais simples, como a observação direta estruturada contínua, ou os mais elaborados, com ênfase na amostragem de trabalho, desenvolvidos pela engenharia de produções ${ }^{(3)}$.

O tempo, no nosso estudo, constitui-se em uma variável importante para mensurar o custo relacionado ao trabalho da enfermeira na realização da Consulta de Enfermagem.

\begin{abstract}
A Assistência de Enfermagem engloba várias atividades, dentre elas a Consulta de Enfermagem que em planejamento de saúde é considerada como uma atividade fim, passível de mensuração, um dado de grande valor para a programação das ações de saúde e, conseqüentemente, para a avaliação das mesmas ${ }^{(5)}$.
\end{abstract}

O Artigo 11, inciso I, alínea “i” da Lei do Exercício Profis-

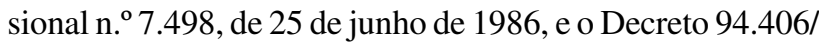
87 legitimam a Consulta de Enfermagem como uma atividade privativa do enfermeiro $^{(6)}$.

O Comitê de Consulta de Enfermagem ${ }^{(a)}$, reunido no XXXI Congresso Brasileiro de Enfermagem, em 1979, definiu Consulta de Enfermagem como:

a atividade diretamente prestada pela enfermeira ao cliente, através da qual são identificados problemas de saúde/ doença e prescritas e implementadas medidas de enfermagem que contribuam à promoção, proteção, recuperação ou reabilitação ao cliente.

\section{A Consulta de Enfermagem é definida como}

o conjunto de ações realizadas pela enfermeira, em uma sucessão ordenada, para conhecer a situação de saúde da clientela e tomar decisões quanto à assistência a ser prestada, visando mudanças favoráveis à saúde ${ }^{(7)}$.

Essas ações objetivam conscientizar o cliente, durante a consulta, levando-o a promover mudanças na sua qualidade de vida, adquirindo hábitos de vida saudáveis.

Na definição estabelecida pela Resolução COFEN-159/96 ${ }^{(6)}$,

a Consulta de Enfermagem é uma atividade privativa do enfermeiro que utiliza componentes do método científico para identificar situação de saúde/doença, prescrever e implementar medidas de enfermagem que contribuam para a promoção, prevenção, proteção da saúde, recuperação e reabilitação do indivíduo, família e comunidade.

(a) Resumo da discussão em Comitê de Especialistas sobre Consulta de Enfermagem, realizada no XXXI CBEn -Fortaleza - Ceará - 1979. 
A Enfermeira, ao atender um indivíduo não hospitalizado, aparentemente sadio ou doente, em tratamento ambulatorial, poderá aplicar o processo de enfermagem e, quando o faz, está realizando uma Consulta de Enfermagem ${ }^{(8)}$.

Diante das colocações acima, depreende-se que a Consulta de Enfermagem é similar ao processo de enfermagem aplicada aos pacientes internados, o que a diferencia é a sua aplicação no contexto ambulatorial.

A aplicação do processo de enfermagem com ênfase no auto-cuidado, nos indivíduos não hospitalizados, propicia aos enfermeiros exercerem sua autonomia profissional, bem como explorarem novas dimensões da profissão(9).

A Consulta de Enfermagem, para ter validade prática, deve proporcionar mudanças favoráveis na saúde do indivíduo $^{(5)}$. Estudos demonstram que a atuação do enfermeiro, por meio da Consulta de Enfermagem, melhora a adesão ao tratamento, acelera o restabelecimento do paciente e, acreditamos, diminui o custo final da assistência.

Para o desenvolvimento da Consulta de Enfermagem, é necessária a observação de alguns fatores, além da metodologia, da eficácia e da capacitação da enfermeira. Segundo pesquisador ${ }^{(5)}$, é necessário formalizar a atividade de Consulta de Enfermagem na instituição como integrante das ações do sistema de prestação de serviços de saúde; e a adequação das normas de atendimento para possibilitar o seu desenvolvimento e opção do cliente para essa atividade.

Em relação ao ambiente físico onde ocorre a Consulta de Enfermagem, é preciso observar as instalações que propiciem a interação enfermeira/paciente, na qual se respeite a privacidade do paciente, sem interrupções de terceiros que possam interromper o elo da comunicação que se está estabelecendo. $\mathrm{O}$ mobiliário e equipamento, para esse atendimento, também devem ser adequados.

Diante do exposto, constatamos que para o desenvolvimento da Consulta de Enfermagem são necessários profissionais capacitados para essa atividade, além de condições materiais e físicas para o seu desenvolvimento. Dessa forma, consideramos importante iniciar estudos que possibilitem mensurar o custo para a sua realização, visando auxiliar o planejamento de recursos a serem utilizados nesse procedimento.

A Resolução COFEN n. ${ }^{\circ} 159 / 96^{(6)}$, que dispõe sobre a Consulta de Enfermagem, normatizando-a, não menciona o tempo que deve ser reservado para a sua realização.

A Portaria n. ${ }^{\circ} 1101$, do Ministério da Saúde ${ }^{(10)}$, de 12 de junho de 2002, que estabelece os parâmetros assistenciais do SUS, preconiza como capacidade de produção para o enfermeiro 03/consultas/hora e para o médico 04/consultas/ hora, não fazendo distinção entre a consulta nova e consulta de seguimento. Ressalta que esses dados podem variar de acordo com convenções sindicais, dissídios coletivos das categorias profissionais ou adoção, pelo gestor, de políticas específicas.

Diante dessas diferentes constatações e por não dispormos de trabalhos referentes a essa temática, optamos por estudar o tempo despendido durante a realização da Consulta de Enfermagem na Seção de Ambulatório do Instituto de Coração (InCor) do Hospital das Clínicas da Faculdade de Medicina da Universidade de São Paulo, para um melhor planejamento da assistência e do agendamento e, ainda, estabelecer um parâmetro para definir o preço.

\section{OBJETIVOS}

- Caracterizar os atendimentos da Consulta de Enfermagem, segundo o grupo de pacientes do programa pré-cirúrgico de coronária, do ambulatório de cirurgia eletiva e as enfermeiras que as realizam.

- Estimar o tempo médio da Consulta de Enfermagem e, com base nele, o correspondente custo para atender pacientes do grupo de coronária.

- Estudar a existência de associação/correlação entre o tempo, o custo referido e as variáveis do programa de consulta de coronária.

\section{METODOLOGIA}

O estudo, do tipo prospectivo, exploratório, descritivocorrelacional, foi desenvolvido no período de 30 de abril a 31 de julho de 2003, na Seção de Ambulatório(AB) do Instituto do Coração (InCor ) do Hospital das Clínicas da Faculdade de Medicina da Universidade de São Paulo. Trata-se de um Hospital público especializado em cardiologia, que atende, além do Sistema Único de Saúde (SUS), pacientes de diversos convênios e pacientes particulares.

A população alvo deste estudo constituiu-se de pacientes pertencentes ao programa pré-cirúrgico, do Ambulatório de Cirurgia Eletiva (ACE), do grupo de coronariopatias, para o qual o desenvolvimento da consulta segue o modelo assistencial denominado Sistemática de Assistência de Enfermagem (SAE), aplicando-se 4 das 6 fases do processo preconizado por Horta $^{(8)}$ : histórico de enfermagem, plano assistencial, prescrição de enfermagem, evolução de enfermagem. A amostra foi constituída de 44 pacientes do grupo de coronariopatias, que foram atendidos em 2 modalidades de Consulta de Enfermagem - nova e de seguimento, sendo 37 em consultas novas e 7 em consultas de seguimento.

O projeto de pesquisa foi encaminhado a Comissão de Ética de Projetos de Pesquisa da Diretoria Clínica do Hospital das Clínicas da FMUSP e para a Comissão Científica e de Ética do Instituto do Coração, sendo aprovada por ambas. 
Para a realização da coleta de dados, foram convidadas as 4 enfermeiras assistenciais da Unidade, que desenvolvem a Consulta de Enfermagem, obtendo-se das mesmas a assinatura no Termo de Consentimento Livre e Esclarecido. As Enfermeiras foram denominadas no estudo de A,B,C e D. Para o levantamento do tempo médio despendido pelas enfermeiras na Consulta de Enfermagem, foi registrado a hora de início e término da consulta, no instrumento de coleta de dados, pelas próprias enfermeiras que as realizaram. A enfermeiras assinalaram também as fases da consulta que tinham realizado, são elas: análise do prontuário, realização do histórico de enfermagem, orientações, solicitações de exames, realização de procedimentos, registro da avaliação/evolução e prescrição e agendamento de retorno e, ainda, se a consulta era nova ou de seguimento.

O levantamento do custo do trabalho da enfermeira, durante a Consulta de Enfermagem, foi realizado baseado na média de tempo gasto e no custo/hora da enfermeira, calculado pela média salarial das profissionais que participaram do estudo. Para o cálculo dos salários das enfermeiras encontramos a média salarial de $\mathrm{R} \$ 4.419,95$. Esse montante foi dividido por 200 horas mensais contratuais. O custo da enfermeira por hora, no InCor, foi em torno de R \$22,09 e de R \$ 0,37 por minuto. O custo da consulta de enfermagem foi calculado multiplicando o tempo médio gasto em minutos pelo custo médio do salário da enfermeira por minuto.

A análise descritiva foi feita sobre as variáveis respostas utilizando distribuições de frequência e as medidas resumo média, desvio padrão, mediana, mínimo e máximo. Na análise inferencial foram utilizados: Teste de Normalidade de Kolmo- gorov - Smirnov, Intervalos de confiança de $95 \%$ para a Média, Teste t-Student, Teste U de Mann Witney, Análise de Variância Clássica, Coeficiente de Correlação Linear de Pearson, Coeficiente de Correlação Ordinal de Sperman e Teste Qui-Quadrado de Associação e Teste de Kruskall-Wallis.

\section{ANÁLISE E DISCUSSÃO DOS RESULTADOS}

Analisando-se o conjunto de Consultas de Enfermagem aos pacientes do programa de coronária obtivemos: das 44 consultas realizadas, $37(84 \%)$ foram de consultas novas e 7 $(16 \%)$, foram de consultas de seguimento.

O exame da Tabela 1 sugere cumprimento pleno das fases 1,3 e 6 tanto nas consultas novas, quanto nas de seguimento e no conjunto das consultas. Vale destacar que a fase 5 não foi cumprida em qualquer um dos tipos de consulta. Ressaltamos, também, que o Histórico de Enfermagem não foi totalmente feito no conjunto das consultas, ou seja, nem nas consultas novas nem nas de seguimento, porque durante a coleta de dados, foram atendidos pacientes que já haviam passado por consulta nova e estavam passando, novamente, por consulta de seguimento. Observamos, também, que em um paciente de consulta de seguimento foi realizado o Histórico de Enfermagem, isso se explica, pois na prática quando no desenvolvimento da primeira consulta na fase da Análise do Prontuário se detecta um problema que implica numa ação de imediato da enfermeira como por exemplo: um exame alterado, faz-se primeiro o encaminhamento a outro profissional. No retorno o paciente volta como consulta de seguimento, onde nessa consulta é realizado o Histórico de Enfermagem.

Tabela 1 - Distribuição das consultas do Programa Coronária segundo as fases cumpridas nas consultas - São Paulo - 2003

\begin{tabular}{|c|c|c|c|c|}
\hline \multirow{2}{*}{ Fase } & \multirow{2}{*}{ Descrição } & \multicolumn{3}{|c|}{ Distribuição } \\
\hline & & Nova & Seg. & Total \\
\hline 1 & Análise do prontuário & 37 & 7 & 44 \\
\hline 2 & Realização do histórico de enfermagem & 37 & 1 & 38 \\
\hline 3 & Orientações & 37 & 7 & 44 \\
\hline 4 & Solicitações de exames & 1 & 1 & 2 \\
\hline 5 & Realização de procedimentos & 0 & 0 & 0 \\
\hline 6 & Registro da avaliação/evolução e prescrição & 37 & 7 & 44 \\
\hline 7 & Agendamento de retorno & 4 & 2 & 6 \\
\hline
\end{tabular}

O exame dos dados da Tabela 2 sugere, para o grupo de consultas novas, predominância de indivíduos em que a consulta cumpriu 4 fases com $33(89,19 \%)$ citações; número médio de fases cumpridas igual a 4,05 (DP=0,53); mediana de 4 e variação de 4 a 6 fases cumpridas. Para o grupo de consultas de seguimento os dados sugerem predominância de consultas em que foram cumpridas 3 fases com $4(57,1 \%)$ citações; número médio de fases cumpridas igual a 3,57 $(\mathrm{DP}=0,79)$; mediana de 3 e variação de 3 a 5 fases cumpridas.
Para o conjunto de consultas os dados sugerem predominância de consultas em que foram cumpridas 4 fases com 34 (77,3\%) citações; número médio de fases cumpridas igual a 4,07( $\mathrm{DP}=0,55)$; mediana de 4 e variação de 3 a 6 . Convém observar que o Teste U de Mann-Whitney mostra evidências de que o número médio de fases no grupo de consulta nova supera o do grupo de consulta de seguimento de forma estatisticamente significativa $(\mathrm{p}=0,041)$. 
Tabela 2 - Distribuição do n. ${ }^{\circ}$ de fases dos atendimentos do Programa Coronária por tipo de consulta - São Paulo - 2003

\begin{tabular}{|c|c|c|c|c|c|c|}
\hline \multirow{3}{*}{ N. ${ }^{\circ}$ fases } & \multicolumn{4}{|c|}{ Tipo de Consulta } & \multirow{2}{*}{\multicolumn{2}{|c|}{ Total }} \\
\hline & \multicolumn{2}{|c|}{ Nova } & \multicolumn{2}{|c|}{ Seguimento } & & \\
\hline & Freq. & $\%$ & Freq. & $\%$ & Freq. & $\%$ \\
\hline 3 & 0 & 0,00 & 4 & 57,1 & 4 & 9,09 \\
\hline 4 & 33 & 89,19 & 2 & 28,6 & 35 & 79,55 \\
\hline 5 & 3 & 8,11 & 1 & 14,3 & 4 & 9,09 \\
\hline 6 & 1 & 2,70 & 0 & 0,0 & 1 & 2,27 \\
\hline TOTAL & 37 & 100,00 & 7 & 100,0 & 44 & 100,00 \\
\hline Média & 4,05 & & 3,57 & & 4,03 & \\
\hline Mediana & 4 & & 3 & & 4 & \\
\hline DP & 0,53 & & 0,79 & & 0,36 & \\
\hline Mín. & 3 & & 3 & & 3 & \\
\hline Máx. & 6 & & 5 & & 6 & \\
\hline
\end{tabular}

O exame dos dados da Tabela 3 sugere predominância de consultas ligadas à enfermeira $\mathrm{D}$ no grupo de consultas novas com $16(43,2 \%)$ citações. No grupo de consultas de seguimento

o predomínio foi de consultas ligadas à enfermeira B com 3 (42,9\%) citações. Para o conjunto de consultas o predomínio coube às consultas ligadas à enfermeira D com 18 (40,9\%) citações.

Tabela 3 - Distribuição das consultas por enfermeiras e tipo de consultas do Programa Coronária - São Paulo - 2003

\begin{tabular}{|c|c|c|c|c|c|c|}
\hline \multirow{3}{*}{ Enfermeira } & \multicolumn{4}{|c|}{ Tipo de Consulta } & \multirow{2}{*}{\multicolumn{2}{|c|}{ Total }} \\
\hline & \multicolumn{2}{|c|}{ Nova } & \multicolumn{2}{|c|}{ Seguimento } & & \\
\hline & Freq. & $\%$ & Freq. & $\%$ & Freq. & $\%$ \\
\hline $\mathrm{A}$ & 3 & 8,1 & 1 & 14,3 & 4 & 9,1 \\
\hline $\mathrm{B}$ & 8 & 21,6 & 3 & 42,9 & 11 & 25,0 \\
\hline $\mathrm{C}$ & 10 & 27,0 & 1 & 14,3 & 11 & 25,0 \\
\hline $\mathrm{D}$ & 16 & 43,2 & 2 & 28,6 & 18 & 40,9 \\
\hline
\end{tabular}

\section{Análise do Tempo}

O exame dos dados da Tabela 4 sugere para o grupo de consultas novas predominância de um tempo de consulta de 28,1 a 46 minutos com 18 (48,6\%) citações; tempo médio igual a 48,91(DP=15,68); mediana de 45 e variação de 10 a 100 minutos. Para as consultas de seguimento os dados sugerem predominância de um tempo de consulta de 10 a 28 minutos com 5 (71,4\%) citações; tempo médio igual a 22,14 (DP=13,80); mediana de 15 e variação de 10 a 50 minutos. Para o conjunto de consultas os dados sugerem predominância de um tempo de consulta de 28,1 a 46 minutos com $19(43,2 \%$ ) citações; tempo médio igual a 44,65 (DP=18,18); mediana de 45 e variação de 10 a 100 minutos. Convém observar que o Teste T-Student para Amostras Independentes mostra evidências de que o tempo médio de atendimento no grupo de consulta nova supera o do grupo de consulta de seguimento de forma estatisticamente significativa $(p=0,000)$. A margem de erro para o tempo médio de atendimento no grupo de consulta nova é 5,14 , isto é, podese inferir, com uma confiança de (95\%), que o tempo médio para o atendimento de consulta nova oscila de 43,77 a 54,05 minutos. A margem de erro para o tempo médio de atendimento no grupo de consulta de seguimento é de 12,71, isto é, pode-se inferir, com uma confiança de (95\%), que o tempo médio de consulta de seguimento oscila entre 9,43 e 34,85 minutos. Para o conjunto de consultas, a margem de erro para o tempo médio de atendimento é 5,48, isto é, pode-se inferir, com uma confiança de $(95 \%)$, que o tempo médio de atendimento oscila entre 39,17 e 50,13 minutos.

\section{Análise de Custo}

Os dados da Tabela 4 também sugerem, para o grupo de pacientes de consultas novas, predominância de um custo de procedimento entre $\mathrm{R} \$ 10,40$ e $\mathrm{R} \$ 16,90$ com 18 (48,6\%) citações; custo médio igual a 18,01 (DP=5,77); mediana de 16,56e variação de $\mathrm{R} \$ 3,68$ a R \$36,82. Para as consultas de seguimento os dados sugerem predominância de um custo do procedimento entre R \$ 3,70 a R \$ 10,30 com 5 (71,4\%) citações; custo médio igual a 8,15 (DP=5,08), mediana de 5,52 e variação de $\mathrm{R} \$ 3,68$ a $\mathrm{R} \$ 18,41$. Para o conjunto de pacientes os dados sugerem predominância de um custo de consulta de $\mathrm{R} \$ 10,40$ a R $\$ 16,90$ com $19(43,2 \%)$ citações; custo médio igual a 16,44 (DP=6,69); mediana de 16,56 e variação entre $R$ \$3,68 a R \$36,82. Convém observar que o Teste T-Student para Amostras Independentes mostra evidências de que o custo médio de atendimento no grupo de consulta nova supera o do grupo de consulta de seguimento de forma estatisticamente significativa $(p=0,000)$. A margem de erro para o custo médio de atendimento no grupo de consulta nova é 1,89 , isto é, pode-se inferir, com uma confiança de $95 \%$, que o custo médio para o atendimento de consulta nova oscila entre R \$16,12 a R \$19,90. A margem de erro para o custo médio de atendimento no grupo de consulta de seguimento é 4,68 , isto é, pode-se inferir, com uma confiança de (95\%), que o custo médio de consulta de seguimento oscila entre $\mathrm{R} \$ 3,47$ a $\mathrm{R} \$ 12,83$. Para o conjunto de consultas a margem de erro para o custo médio de atendimento é de 2,02, isto é, pode-se inferir, com uma confiança de (95\%), que o custo médio de atendimento oscila entre $\mathrm{R} \$ 14,42 \mathrm{a} \mathrm{R} \$ 18,45$. 
Tabela 4 - Distribuição das consultas do Programa Coronária por tempo, custo de atendimento e tipo de consulta - São Paulo - 2003

\begin{tabular}{|c|c|c|c|c|c|c|}
\hline \multirow{3}{*}{$\begin{array}{c}\text { Tempo } \\
(\min )\end{array}$} & \multicolumn{4}{|c|}{ Tipo de Consulta } & \multicolumn{2}{|c|}{ Total } \\
\hline & \multicolumn{2}{|c|}{ Nova } & \multicolumn{2}{|c|}{ Seguimento } & \multirow[t]{2}{*}{ Freq. } & \multirow[t]{2}{*}{$\%$} \\
\hline & Freq. & $\%$ & Freq. & $\%$ & & \\
\hline 10,0 a 28 & 1 & 2,7 & 5 & 71,4 & 6 & 13,6 \\
\hline 28,1 a 46 & 18 & 48,6 & 1 & 14,3 & 19 & 43,2 \\
\hline 46,1 a 64 & 12 & 32,4 & 1 & 14,3 & 13 & 29,5 \\
\hline 64,1 a 82 & 5 & 13,5 & 0 & 0,0 & 5 & 11,4 \\
\hline 82,1 a 100 & 1 & 2,7 & 0 & 0,0 & 1 & 2,3 \\
\hline TOTAL & 37 & 100,0 & 7 & 100,0 & 44 & 100,0 \\
\hline Média & 48,91 & & 22,14 & & 44,65 & \\
\hline Mediana & 45,00 & & 15,00 & & 45,00 & \\
\hline DP & 15,68 & & 13,80 & & 18,18 & \\
\hline Min. & 10,00 & & 10,00 & & 10,00 & \\
\hline Máx. & 100,00 & & 50,00 & & 100,00 & \\
\hline IC $95 \%$ & 5,14 & & 12,71 & & 5,48 & \\
\hline Custo & Freq. & $\%$ & Freq. & $\%$ & Freq. & $\%$ \\
\hline 3,7 a 10,3 & 1 & 2,7 & 5 & 71,4 & 6 & 13,6 \\
\hline 10,4 a 16,9 & 18 & 48,6 & 1 & 14,3 & 19 & 43,2 \\
\hline 17,0 a 23,6 & 12 & 32,4 & 1 & 14,3 & 13 & 29,5 \\
\hline 23,7 a 30,2 & 5 & 13,5 & 0 & 0,0 & 5 & 11,4 \\
\hline 30,3 a 36,8 & 1 & 2,7 & 0 & 0,0 & 1 & 2,3 \\
\hline TOTAL & 37 & 100,0 & 7 & 100,0 & 44 & 100,0 \\
\hline Média & 18,01 & & 8,15 & & 16,44 & \\
\hline Mediana & 16,56 & & 5,52 & & 16,56 & \\
\hline DP & 5,77 & & 5,08 & & 6,69 & \\
\hline Mín. & 3,68 & & 3,68 & & 3,68 & \\
\hline Máx. & 36,82 & & 18,41 & & 36,82 & \\
\hline IC $95 \%$ & 1,89 & & 4,68 & & 2,02 & \\
\hline
\end{tabular}

Os dados da Tabela 5 mostram evidências estatísticas de que o Tempo de Consulta e o correspondente Custo apresenta correlação positiva estatisticamente significativa com o número de fases $(\mathrm{r}=0,369, \mathrm{p}=0,014)$. O resultado indica que consultas que consomem maior número de fa- ses tendem a consumir maior tempo e, conseqüentemente, maiores custos. Evidencia que o tempo (e o correspondente custo) não apresenta correlação estatisticamente significativa com a idade, o número de diagnósticos ou número de fase.

Tabela 5 - Correlação entre as variáveis escalares do estudo para o grupo de pacientes do Programa Coronária - São Paulo - 2003

\begin{tabular}{|c|c|c|c|c|c|}
\hline & Tempo_M & Idade & N Diag & N Fases & Custo \\
\hline Tempo_M & 1,000 & & & & \\
\hline Idade & 0,063 & 1,000 & & & \\
\hline N Diag & 0,230 & 0,139 & 1,000 & & \\
\hline N Fases & $0,369^{*}$ & 0,026 & 0,055 & 1,000 & \\
\hline Custo & 1,000 & 0,063 & 0,230 & $0,369 *$ & 1,000 \\
\hline
\end{tabular}

*Correlação estatisticamente significativa.

Oexame da Tabela 6 oferece evidência estatística de correlação estatisticamente significativa entre Tempo e o correspondente custo) e a Enfermeira que realizou o procedimento. Acomparação 2 a 2 do tempo (e custo) médio das enfermeiras revelou que os tempos (e custo) médio de atendimento das enfermeiras A, B e $\mathrm{C}$ não diferem de forma estatisticamente significativa, todavia, revelou que a enfermeira $\mathrm{D}$ tende a apresentar um tempo (e custo) médio significativamente superior ao das enfermeiraAeC.

Tabela 6 - Teste de Kruskall-Wallis para a correlação entre tempo, custo das enfermeiras- São Paulo - 2003

\begin{tabular}{lccc} 
& Enfermeira & N & Posto Médio \\
\hline \multirow{2}{*}{ TEMPO } & A & 18 & 31,97 \\
& B & 23 & 46,74 \\
C & 25 & 34,38 \\
CUSTO & D & 16 & 55,81 \\
& Total & 82 & 31,97 \\
& A & 18 & 46,74 \\
& B & 23 & 34,38 \\
& D & 16 & 55,81
\end{tabular}




\section{CONSIDERAÇÕES FINAIS}

O dimensionamento dos recursos humanos, a adequação dos materiais necessários, o tempo despendido para a realização dos procedimentos e, ainda, os custos, são fontes de preocupação das enfermeiras, principalmente, daquelas que exercem atividades gerenciais. Essa preocupação procede porque se sabe que a qualidade da assistência está atrelada à adequação desses recursos, pois, normalmente, eles são escassos.

Determinar qual o tempo despendido pela enfermeira na realização da Consulta de Enfermagem, nos orienta para o dimensionamento de pessoal necessário para o desenvolvimento deste procedimento (lembrando que este deve estar baseado na filosofia estabelecida pela instituição e sustentado pelo referencial teórico), organizar a programação de agendamento melhor atender o paciente e também prever o custo do trabalho da enfermeira nesse procedimento.

A Consulta de Enfermagem consumiu um tempo médio de aproximadamente 48,91 minutos para as consultas novas do grupo de coronária, e o custo da enfermeira foi de aproximadamente $\mathrm{R} \$ 18,01$. As consultas de seguimento levaram, em média, 22,14 minutos, e o custo da enfermeira girou em torno de R $\$ 8,15$. Se compararmos com o que o SUS reembolsa $-\mathrm{R} \$ 2,04$ constatamos que o valor repassado pelo SUS fica muito aquém do cálculo efetuado.

\section{REFERÊNCIAS}

(1) Drumond MF, Stoddart GL, Torrance GW. Methods for economic evaluation of health care programmes. Oxford: Oxford University Press; 1992.

(2) Martins E. Contabilidade de custos: inclui ABC. $7^{a}$ ed. São Paulo: Atlas; 2000.

(3) Mello MC. Estudo do tempo no trabalho da enfermagem: construção de instrumento de classificação de atividades para implantação do método amostragem do trabalho [dissertação]. São Paulo: Escola de Enfermagem da USP; 2002.

(4) Gaither N, Frazier G. Administração da produção e operações. Trad. de José Carlos Barbosa dos Santos. $8^{\mathrm{a}}$ ed. São Paulo: Pioneira Thomsom Learning; 2001.

(5) Araújo EC. Assistência de enfermagem a pacientes externos. Rev Bras Enferm. 1979;32(4):385-95.

(6) Conselho Federal de Enfermagem. Resolução COFEN n. 159, de 19 de abril de 1993. Dispõe sobre a consulta de enfermagem. In: Conselho Regional de Enfermagem. Documentos básicos de enfermagem: enfermeiros, técnicos, auxiliares. São Paulo: COREn-SP; 1997. p. 101-2.
Esta realidade nos leva a refletir sobre a necessidade de gerenciarmos custos, porque em todas as literaturas consultadas a respeito de custo, que foram realizadas em hospitais governamentais, foi constatado que o repasse efetuado pelo SUS para os procedimentos não cobrem os gastos.

Este estudo permitiu refletir sobre a importância de as enfermeiras que gerenciam unidades conhecerem o tempo gasto e o custo dos procedimentos, pois, é mediante comprovações fundamentadas e baseadas em evidências científicas que se justifica a necessidade de recursos. Dessa forma, acredita-se ser possível melhorar a qualidade da assistência e as condições de trabalho das profissionais, considerando sempre o ato do cuidar.

Como já dissemos, não é nossa intenção com este estudo preconizarmos o tempo a ser despendido por enfermeiras na realização da Consulta de Enfermagem e nem definir um padrão.

Sabemos que este estudo é um primeiro desafio para muitos outros que poderão ser realizados, uma vez que não encontramos na literatura pesquisas sobre este enfoque.

Considerando que a Consulta de Enfermagem pode beneficiar o paciente no seu contexto biopsicossocial, e também a experiência adquirida no decorrer desse estudo, propomos futuramente, uma análise da Consulta de Enfermagem com uma visão voltada para o Custo Benefício e do Custo Efetividade.

(7) Campedelli MC. Consulta de enfermagem ao binômio mãefilho, análise das condutas de enfermagem [tese]. São Paulo: Faculdade de Saúde Pública da USP; 1986.

(8) Horta WA. Processo de enfermagem. São Paulo: EPU/EDUSP; 1979.

(9) Melleiro MM. A consulta de enfermagem no cenário do sistema de assistência de enfermagem. In: Cianciarullo TI, Gualda DMR, Melleiro MM, Anabuki MH, organizadoras. Sistema de assistência de enfermagem: evolução e tendências. São Paulo: Ícone; 2001. p. 279-92.

(10) Brasil. Ministério da Saúde. Portaria n. 1101, de 12 de junho de 2002. G.1) Capacidade de produção, em consultas, de alguns recursos humanos na área de saúde [on line]. Brasília; 2002. Disponível em: <http://portalweb01.saude.gov.br/ saude/buscar.cfm $>$ [Acesso em 22 nov 2003]. 